\title{
Multiple Metachronous Granular Cell Tumors of the Gastrointestinal Tract Within a Single Patient: a Case Report and Review of the Literature
}

\author{
Jeffrey M. Sutton MD ${ }^{1, *}$, Jonathan Tobias $\mathrm{MD}^{2}$, Ian M. Paquette $\mathrm{MD}^{3}$ \\ ${ }^{1}$ University of Cincinnati Medical Center Department of Surgery 231 Albert Sabin Way ML 0558, Room SRU-1588 \\ Cincinnati, OH 45267 \\ ${ }^{2}$ Greater Cincinnati Pathologists 2134 Auburn Avenue \\ ${ }^{3}$ University of Cincinnati Medical Center Department of Surgery Division of Colon and Rectal Surgery, \\ 2123 Auburn Avenue \# 524, Cincinnati, OH 45219 \\ *Corresponding Author: jmsutton@gmail.com
}

Copyright (c) 2013 Horizon Research Publishing All rights reserved.

\begin{abstract}
Introduction: Granular cell tumors are a rare form of soft tissue tumor often arising from the skin and subcutaneous tissues of the head, trunk, or extremities, although they rarely may arise within the colon or gastrointestinal tract.Case Report:We present a case of a 28 year-old female who initially presented with recurrent granular cell tumors of the stomach treated with endoscopic polypectomy. She eventually developed a metachronous lesion within the ascending colon and underwent a segmental colectomy. We present her treatment course, pathological findings, and review of the literature of this unique condition.
\end{abstract}

Keywords Granular Cell Tumor, Abrikossoff Tumor, Metachronous, Recurrence, Colectomy

\section{Case Presentation}

An otherwise healthy 28-year-old African American female presented to her gastroenterologist in 2006 for evaluation of vague gastrointestinal symptoms of abdominal cramping, burning, and epigastric pain. She underwent anesophagogastroduodenoscopy (EGD) with forceps biopsy of a "mucosal prominence probably representing a gastric polyp" within the gastric fundus near the cardioesophageal junction. The pathology of the biopsy returned as a sub-centimeter granular cell tumor (GCT) (Figure 1). The remainder of her EGD, including esophageal visualization and antral biopsy for Helicobacter pylori, was negative.

She returned for a follow-up EGD in 2008, which demonstrated recurrence of the GCT in the fundus of the stomach. The lesion was resected by snare polypectomy using a saline lift technique. Histology revealed a recurrent GCT. Immunostains were positive for S-100 and negative for pankeratin (AE1+AE3/CAM5.2) and CD117 (c-kit), a pattern consistent with granular cell tumors.

A third EGD performed in 2010 for evaluation of symptom recurrence again demonstrated a 6 × 5 x $5 \mathrm{~mm}$ firm solid white well-circumscribed submucosal nodule along the gastric fundus. This nodule was again resected via snare polypectomy, and pathology confirmed the diagnosis of recurrent GCT.

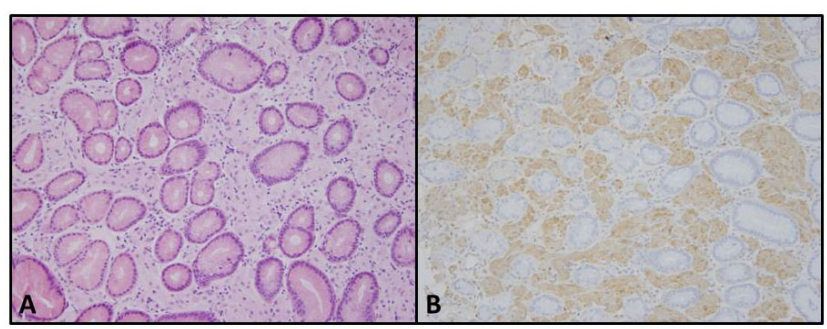

Figure 1. Stomach polyp from 2006. A) H\&E (200x) of granular cell tumor. The lamina propria of the gastric antral mucosa is expanded by a proliferation of polygonal cells with small nuclei, inconspicuous nucleoli, and abundant granular cytoplasm. No cellular atypia or mitotic figures are seen. B) S-100 immunohistochemical stain $(200 \mathrm{x})$ is positive within the granular cell tumor cells.

Given her family history of colon cancer in a first-degree relative and multiple bouts of colon cancer in second-degree relatives, in addition to her recurrent GCT, she underwent colonoscopy in early 2012 at the age of 34 . A submucosal nodule within the cecum at the base of the ileocecal valve was tattooed and biopsied, with pathology consistent with GCT. She was subsequently referred to a colorectal surgeon for further evaluation. After discussion with the patient, her surgeon elected to perform a segmental resection given the patient's personal history of recurrence and given the small potential for malignant degeneration of GCTs. Following a negative EGD, she underwent a laparoscopic right hemicolectomy. Final pathology confirmed complete 
excision of a $9 \mathrm{~mm}$ submucosal nodule adjacent to the ileocecal valve histologically consistent with a GCT (Figure2).No malignancy was identified within the nodule nor within the lymph nodes harvested at the time of surgery. Her postoperative course was uneventful.

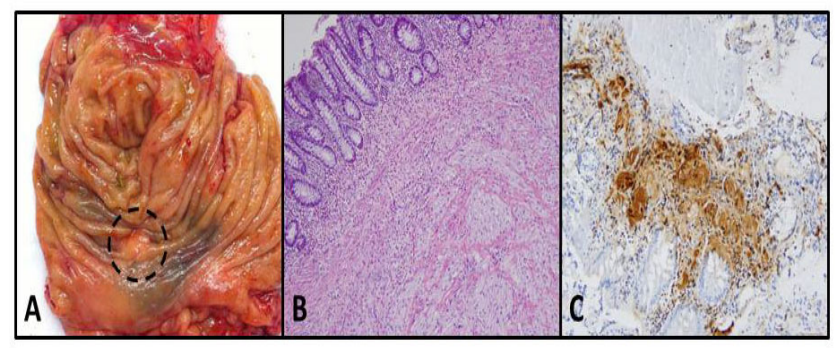

Figure 2 .Cecal polyp from 2012. A) Gross specimen, right hemicolectomy. Note the $9 \mathrm{~mm}$ submucosal nodule the cecum adjacent to the ileocecal valve. This nodule was a pathologically confirmed granular cell tumor. B) H\&E (100x) demonstrating recurrent granular cell tumor. Note the poorly circumscribed submucosal tumor comprised of polygonal cells with small nuclei, inconspicuous nucleoli, and abundant granular cytoplasm. The tumor cells focally involve the overlying mucosa as well as extending into the superficial portion of the underlying muscularis propria. No cellular atypia was observed. C) S-100 immunohistochemical stain (200x) demonstrating positive tumor cells in brown.

\section{Discussion}

First described by Abrikossoff in 1926 [1], granular cell tumors are a rare family of soft tissue tumors. Although they were originally thought to resemble myosarcoma-like tumors, GCTs currently favor a Schwann-cell origin. More recent studies [2] have found close anatomic association with nerves as well as positive staining for S-100 protein and neuron-specific enolase, both markers for neural derivation. Additional histologic findings consistent within GCTs include a proliferation of bland round-to-ovoid cells with abundant eosinophilic granular cytoplasm organized into distinct nests; small, pyknotic, and centrally located nuclei; and the accumulation of periodic acid-Schiff positive, diastase-resistant lysosomal granules within the cytoplasm [3]. Significant Ki-67 staining, mitotic figures, or tumor necrosis are rarely observed. Notably, histology alone is a poor predictor of biological behavior within GCTs.

These tumors tend to cluster in patients between the fourth and sixth decades, however reports exist identifying GCTs in patients ranging from 16 to 71 years of age. Most reports agree that GCTs demonstrate a slight female predominance and appear to be more common within the African American race [4].

Though they may occur at virtually any site of the body, GCTs demonstrate a predilection for the skin and subcutaneous tissue of the surface of the head, neck, trunk, breast tissue, and extremities [5]. Additionally, they may develop within the oral cavity, specifically along the tongue. Visceral lesions within the mucosa and submucosa of the gastrointestinal tract are comparatively rare, comprising only $5-8 \%$ of all GCTs [4]. Of these tumors, the vast majority occur within the esophagus followed by the large intestine (ascending $>$ transverse $>$ descending). Localization within the stomach is a rare occurrence with only approximately 30 independent reports within the literature [5].

Dependent upon the size and location of the lesion(s), GCTs can produce vague gastrointestinal symptoms of abdominal pain, nausea, or obstructive-like symptoms. However, they typically remain asymptomatic until identified incidentally on routine endoscopic evaluation or workup for other symptoms [6]. Grossly, GCTs appear as small, firm, yellow, well-circumscribed round nodules within the submucosa covered by normal-appearing mucosa or epithelial tissue. They are often misdiagnosed as small lipomas upon visualization [6].

Such a significant proportion of GCTs exhibit a benign course that malignant GCTs are considered the rare exception to the rule. Approximately $2 \%$ of all GCTs are thought to harbor malignant characteristics, and even fewer ever metastasize [7]. Though it remains difficult to always accurately distinguish between benign and malignant GCTs, Khansur and colleagues [8] identified 6 histological criteria associated with an increased risk of malignancy: increased mitotic rate (i.e., 2 or greater mitoses per 10 high-power fields at x200 magnification), tumor cell spindling, presence of vesicular nuclei with large nucleoli, a high nuclear to cytoplasmic ratio, pleomorphism, and tumor necrosis. A follow-up study [9] further delineated that tumors exhibiting only focal pleomorphism could be classified as benign, while tumors exhibiting one or two criteria were classified as atypical lesions. Possessing three or more of the abovementioned criteria earned the classification of malignant. Although the vast majority of GCTs are solitary, they may occur synchronously as multiple lesions in approximately $10-15 \%$ of patients [10]. Synchronous tumors are most common in the setting of gastric GCT, with greater than half of all gastric GCTs occurring synchronously with additional gastric lesions and/or concurrent esophageal GCTs [6]. Published case reports [11-13] discuss the synchronous potential of simultaneous GCTs isolated exclusively to the colon; within multiple sites of the esophagus and stomach; involving the colon, appendix, and mesentery; and even synchronous involvement of the perianus and chest wall. However, to our knowledge, this report is the first publication of multiple metachronous GCTs presenting within the stomach and colon within the same patient over a multiple year timeframe.

\section{Treatment}

Precise guidelines regarding appropriate treatment of GCTs have yet to be established, given the relative rarity of occurrence. Practice patterns range from conservative endoscopic or colonoscopic resection with strict endoscopic follow up [10] to aggressive surgical intervention $[12,14]$. While endoscopic submucosal resection has typically been favored in the setting of isolated esophageal lesions, 
increasing reports are demonstrating similar success with endoscopic mucosal resection of GCTs within the colon as well [10]. Endoscopic ultrasound and endoscopic removal of GCTs has been suggested as the treatment of choice for smaller lesions less than $2 \mathrm{~cm}$ not involving the muscularis propria [15]. Alternatively, surgical resection is typically favored if the tumor demonstrates the abovementioned features suggestive for malignancy, or when multiple lesions exist synchronously. Case reports have discussed opting for a gastrectomy in a patient with 7 simultaneous gastric GCTs [12] or for a total colectomy in a patient with 52 pan-colonic GCTs [14]. Patients should be evaluated on a case-by-case basis, and treatment decisions should take into account the low risk of malignant degeneration but relatively high risk of local recurrence.Close follow-up is recommended for patients who remain at high-risk for developing associated neoplasms or who demonstrate evidence of multiple lesions or tumor recurrence.

\section{REFERENCES}

[1] A. Abrikossoff. Ausgehend von der quergestreiften willkurlichen Muskulatur. Virchows Arch Pathol Anat 1926;260:215-33.

[2] I.S. Seo, B. Azzarelli, T.F. Warner, M.P. Goheen, G.E. Senteney. Multiple visceral and cutaneous granular cell tumors. Ultra-structural and immunocytochemical evidence of Schwann cell origin. Cancer 1984;53:2104-2110.

[3] J.R. Goldblum, T.W. Rice, G. Zuccaro, J.E. Richter. Granular Cell Tumors of the Esophagus: A Clinical and Pathologic Study of 13 Cases. Ann Thorac Surg 1996;62:860-5.

[4] D. Pertile, S. Scabini, E. Romairone, R. Scordamaglia, E. Rimini, V. Ferrando. Gastric Abrikosoff tumor (granular cell tumor): case report. G Chir 2010;31(10):433-434.
[5] R. Patti, P.L. Almasio, G.D. Vita. Granular cell tumor of stomach: A case report and review of literature. World $\mathrm{J}$ Gastroenterol 2006;12(21):3442-3445.

[6] L.M. Wang, R. Chetty. Selected Unusual Tumors of the Stomach: A review. Internat J Surg Path 2012;20(1):5-14.

[7] J. Chen, L. Wang, J. Xu, T. Pan, J. Shen, W. Hu, X. Yuan. Malignant granular cell tumor with breast metastasis: a case report and review of the literature. Oncology Letters 2012;4:63-66.

[8] T. Khansur, L. Balducci, M. Tavassoli. Granular cell tumor. Clinical spectrum of the benign and malignant entity. Cancer 1987;60:220-222.

[9] J.C. Fanburg-Smith, J.M. Meis-Kindblom, R. Fante, L.G. Kindblom. Malignant granular cell tumor of soft tissue: Diagnostic criteria and clinicopathologic correlation. Am J Surg Pathol 1998;22:779-794.

[10] G.B. Rossi, M. de Bellis, P. Marone, A. De Chiara, S. Losito, A. Tempesta. Granular cell tumors of the colon: report of a case and review of the literature. J Clin Gastroenterol 2000; 30:197-199.

[11] J.B. Soares, A. Machado, R. Goncalves. Multiple granular cell tumor of the colon. Endoscopy 2010;42:E158-E159.

[12] H. Maekawa, T. Maekawa, K. Yabuki, K. Sato, Y. Tamazaki, K. Kudo, R. Wada, M. Matsumoto. Multiple esophagogastric granular cell tumors. J Gastroenterol 2003;38:776-780.

[13] H. Saleh, M. El-Fakharany, M. Frankle. Multiple Synchronous Granular Cell Tumors Involving the Colon, Appendix, and Mesentery: A Case Report and Review of the Literature. J Gastrointestin Liver Dis 2009;18(4):475-478.

[14] C.R. Melo, I.S. Melo, F. Schmitt, R. Faqundes, D. Amendola. Multicentric granular cell tumor of the colon: report of a patient with 52 tumors. Am J Gastroenterol 1993;88(10):1785-1787.

[15] D.L. Lowe, A.J. Chaudhary, J.R. Lee, S.M. Chamberlain, R.R. Schade, U. Cuartas-Hoyos. Four Cases of Patients with Gastrointestinal Granular Cell Tumors. Southern Medical Journal 2007;100(3):298-300 